\title{
ALTERAÇÕES NA DISTRIBUIÇÃO DA LUZ NATURAL CAUSADOS PELOS AGRUPAMENTOS DE ALUNOS EM SALAS DE AULA
}

BEDOYA JARAMILLO, Carolina (1); RUTTKAY PEREIRA, Fernando O.(2)

(1) Universidade Federal de Santa Catarina, e-mail: carobedoya107@gmail.com, (2) Universidade Federal de Santa Catarina, e-mail: feco@arq.ufsc.br

\begin{abstract}
RESUMO
É reconhecido que o aproveitamento da luz natural em escolas é uma das estratégias mais importantes no correto agenciamento dos recursos naturais disponíveis. Por sua vez, a distribuição da luz natural nos espaços internos está sujeita à forma como os usuários ocupam este espaço. As normativas locais e internacionais estabelecem iluminâncias médias de 300lux para salas de aula, mais não especificam se este valor considera a ocupação dos estudantes e como eles interferem na distribuição da iluminação dentro da sala de aula. Não considerar a distribuição luminosa nestes espaços, nos quais os estudantes permanecem a maior parte do tempo, fazem diferentes atividades, que envolvem diferentes agrupamentos, pode gerar avaliações imprecisas. Este estudo de mestrado tem como objetivo avaliar a influência de diferentes tipos de agrupamentos de estudantes em salas de aula, iluminadas naturalmente, e comparar com uma sala de aula vazia. Foram realizadas as simulações computacionais estáticas e dinâmicas de uma Sala de aula hipotética para avaliar a distribuição da luz no dia. O Plug-in DIVA do Rhinos foi usado para calcular a incursão solar no espaço, em dois períodos do ano, durante o horário escolar. O software APOLUX permitiu avaliar a iluminação natural sob diferentes condições de céu em diferentes períodos do ano. Como resultados parciais, este trabalho mostra que, apesar da presença de estudantes nos diferentes tipos de agrupamentos, a distribuição da iluminação no plano de trabalho quando o espaço esta ocupado é levemente inferior as exigências da norma, quando comparadas com a uma sala de aula vazia. Também evidencia que independente do número de estudantes que se encontram numa sala de aula, a maior influência está no tipo de agrupamento, postura e posição dos estudantes em relação às fontes de luz - as aberturas. Este estudo pode servir como método de análise para outros espaços que possuam qualidades de permanência significativas e que necessitam atingir exigências luminosas básicas. Palavras-chave: Iluminação natural, Iluminação em Salas de Aula, Agrupamento de estudantes.
\end{abstract}

\begin{abstract}
It is acknowledged that the use of daylight in schools is one of the most important strategies in the correct employment of the available natural resources. Meanwhile, the distribution of natural light in interior spaces is subject to how its users occupy this space. Local and international regulations establish an average illuminance of 300lux for classrooms, but do not specify whether this value considers the occupation of the students and how they interfere with the distribution of the illuminance inside the classroom. To not consider the light distribution in these spaces, where students spend most of their time, doing different activities and interacting in different ways of grouping, can lead to imprecise evaluations. This study masters aims to evaluate the influence of different groups of students, in naturally lighted classrooms, and compare their light distribution with the distribution in an empty classroom. Static and Dynamic computer simulations of a hypothetical classroom were performed to evaluate the interior light distribution during the day. Rhino's plug-in, DIVA, was used to calculate the solar incursion in the space, in two periods of the year, during school hours. The software APOLUX evaluated the natural lighting under different sky conditions in different periods of the year. As partial results, this paper shows that,
\end{abstract}


despite the presence of students in different types of groupings, the distribution of illumination on the working plane, when the space is occupied, is slightly lower than the requirements of the standard, compared with an empty classroom. It also shows that regardless of the number of students in the classroom, the greatest influence is the type of grouping, posture and position of students in relation to the light source - the openings. This study can serve as a method of analysis for other spaces that have significant periods of permanence and that need to achieve basic light requirements.

Keywords: Daylighting, Daylighting in Classrooms, Groupings of students.

\section{INTRODUÇÃO}

Os estudos de iluminação natural frequentemente relatam sobre a influência que esta tem nas pessoas (no controle do relógio biológico) e nos espaços (disponibilidade de luz ao interior). Porém é difícil encontrar estudos que falem como a presença dos usuários dentro dos espaços interfere na distribuição da iluminação natural. Espaços como as salas de aula, devem considerar, além da presença dos estudantes, a capacidade de ocupação, tipos de agrupamentos para diversas atividades e condições ambientais adequadas visando melhoria do desempenho acadêmico dos estudantes. Existem diferentes pesquisas que estudam a influência da luz natural no desempenho escolar dos estudantes. Estudos desenvolvidos no Canadá por Hathaway et al., (1992) constatam que a presença da luz natural nas salas de aula garante uma aprendizagem mais rápida, maior desenvolvimento físico; e adequadas condições de conforto visual devido à interação dos alunos com as janelas. Em Califórnia, o Heschong Mahone Group, (1999), demostram a relação uniforme e estatisticamente positiva entre a presença da luz natural e melhores notas dos estudantes. No caso Europeu, são aprofundados estudos nas salas de aula do futuro por Fotios, Ramasoot, (2009). Os autores afirmam que é necessário ter um balanço entre a luz admitida pelas janelas e a luz emitida pelos computadores. Observa-se como os estudos vão encaminhados nas afetações da luz no sistema visual e físico das crianças, mais não consideram a ocupação deles como um fator que altere as condições luminosas da sala de aula.

Boa parte de nossa infância e adolescência se dá na escola, já que um $50 \%$ da vida é desenvolvida dentro da sala de aula. Dentro da sala de aula, Segundo Azevedo, (2002), as necessidades espaciais das crianças em idade pré-escolar diferem das necessidades dos adolescentes; sua relação com o espaço é fluída, utilizam menos os recursos verbais e estão abertos aos estímulos visuais e auditivos (LYNCH, 1995). No caso dos adolescentes, "tendem a associar-se em grupos" e para isso necessitam de espaços apropriados onde possam conviver e manifestar-se" (IBAM, 1996). As alterações na distribuição da iluminação na sala de aula causada pela ocupação, estão relacionadas com: o crescimento dos alunos ("growth spurt" na adolescência) e com fatores complementares como os tipos de agrupamentos, posição da janela na sala e as necessidades de iluminação para um ótimo desempenho da tarefa visual.

Na Colômbia, segundo a NTC 4595 (MEN COLÔMBIA, 2006) no item 7.2 que trata sobre "Conforto Visual" é feita referência à orientação das janelas (perpendiculares ou do lado esquerdo do quadro) e a área das aberturas (1/5 da área do piso), com as quais se busca garantir distribuição e uniformidade luminosa e evitar a exposição à radiação direta. No entanto, esta norma não contempla a disposição dos estudantes e os diferentes tipos de agrupamentos dentro da sala de aula, de acordo com sua atividade, e como estes podem afetar as condições de iluminação. Por sua vez, no caso do Brasil, o Caderno Técnico 4, (FUNDAESCOLA 2002), recomenda a percentagem de janela de acordo com a área do piso e a localização das aberturas em relação ao quadro-giz (1/5 da área do piso ou mais, aberturas do lado esquerdo). Além disso, diferentemente da norma 
colombiana, recomenda opções de prática pedagógica, onde existam soluções arquitetônicas que possibilitem diversas formas de arranjo do mobiliário, de modo a permitir organização em pequenos grupos, em círculo, projeções, aulas expositivas e outras mais, com desembaraçada movimentação dos alunos.

Em termo de iluminância, quando se fala dos níveis de necessários para realizar uma tarefa visual, as normativas locais e internacionais, por exemplo, a NBR ISO/CIE 8995, (2013), estabelecem iluminâncias médias de 300lux para salas de aula. Contudo, as normativas não especificam se este valor considera a ocupação dos estudantes. Portanto, garantir níveis de iluminância mínimos numa sala de aula sem considerar a movimentação de seus estudantes, remete avaliar a distribuição da iluminação de forma incompleta, já que adiciona uma variável não presente em um espaço vazio.

\section{OBJETIVO}

Este estudo tem como objetivo avaliar a influência de diferentes tipos de agrupamentos de estudantes em salas de aula, iluminadas naturalmente, e comparar com uma sala de aula vazia.

\section{MÉTODO}

\subsection{Objeto de Estudo}

O objeto de estudo foi uma Sala de aula hipotética modelada com 7,5m de comprimento, $8,1 \mathrm{~m}$ de largura e 3,0m de pé direito, com uma proteção solar horizontal na verga da abertura com $0,60 \mathrm{~m}$ de largura (Ver figura 1). Cabe salientar que a sala cumpre com a área útil por aluno (36 alunos), área obrigatória para iluminação e localização da abertura como é descrito no Caderno Técnico 4. Foram considerados 4 tipos de agrupamentos: Agrupamento de Sala Vazia (AVazia), Agrupamento Fileiras (AFileiras), Agrupamento Grupos (AGrupos) e Agrupamento Mesa Redonda (AMesa $R)$.

\section{Figura 1 - Sala de Aula hipotética e os Tipos de Agrupamentos}

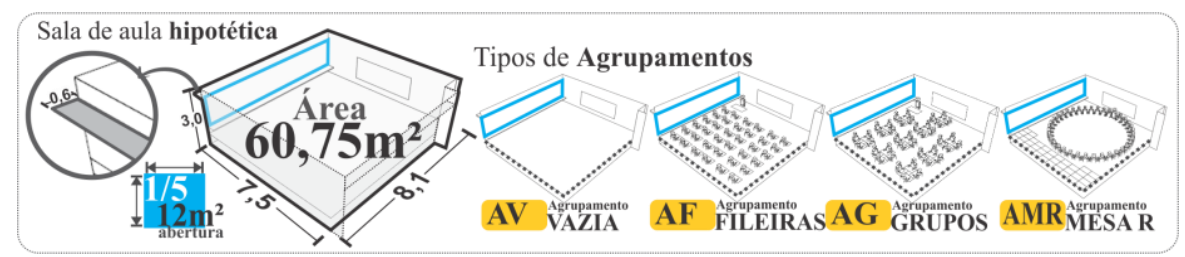

Fonte: dos autores

\subsection{Simulação dos parâmetros Dinâmicos}

A simulação dinâmica faz uso das unidades métricas baseadas no clima que derivam-se de perfis anuais em intervalos de tempo (hora/minuto). Existem diversos programas computacionais que permitem este tipo de simulações: DIVA e Daysim são alguns deles. Estes programas computacionais se encontram baseados em algoritmos de Radiance e fazem uso de arquivos climáticos. No presente estudo foram realizadas simulações dinâmicas no Plug-in DIVA do Rhino, para avaliar a influência da insolação dentro da sala durante dois períodos do ano e dois períodos horários escolares. No 
Programa Rhinoceros/DIVA foi escolhida a localização (com arquivo climático EPWSWERA para a cidade de Florianópolis), a quantidade de pontos e seu espaçamento à altura do plano de trabalho de $0,60 \mathrm{~m}$, os materiais (para os alunos foi assumido material opaco que cumpria com as caraterísticas do material GenericInteriorWall_50 aplicado nas paredes, moveis e o quadro), os parâmetros de Radiance por default (-ab 2 -ad 1000 -as 20 -ar 300 -aa 0.1). Foram avaliados 4 tipos de agrupamentos (Sala Vazia, Filieiras, Grupos e Mesa Redonda), nos meses de Março (03 01/03 30) e Agosto, (08 01/08 30), durante os períodos horários: de manhã $(08$ 12) e de tarde (12 16) nas 4 orientações resultando num total de 64 simulações (Ver Quadro 1)

\section{Quadro 1 - Parâmetros inseridos no Plug-in DIVA}

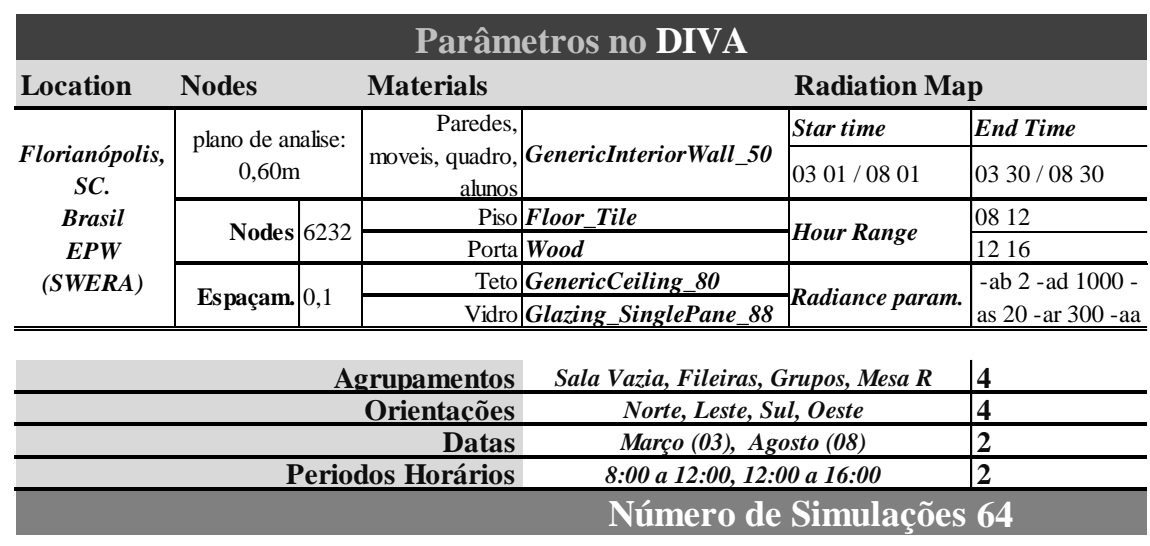

Fonte: dos autores

\subsection{Simulação dos parâmetros Estáticos}

A simulação estática permite avaliações para um local, data e hora específica, de acordo com o tipo de céu predominante. Existem diferentes programas computacionais que permitem este tipo de simulações: Lighttool, Dialux e APOLUX, são alguns deles. No presente estudo foram realizadas simulações estáticas no APOLUX para avaliar a distribuição da luz na sala sob diferentes condições de céu, períodos do ano e horários. De acordo com o objetivo do estudo, não foi usada a simulação dinâmica da iluminação, pois esta supõe uma posição fixa dos estudantes ao longo do tempo descartando a possibilidade dos alunos se movimentarem dentro da sala.

A sala de aula foi exportada para o Módulo Fractal do APOLUX, onde foram escolhidas as propriedades óticas dos materiais, especificando o "vidro" como material transmissor difuso, a fim de garantir maior controle da luz exterior que não consegue ser controlada pela proteção solar. Foi determinado o Fator de Divisão ou fraccionamento para cada superfície da sala, considerando o maior fraccionamento no "plano de trabalho" (20), já que nele foi aprofundada a análise da distribuição da iluminação natural. A área máxima do fraccionamento do modelo foi de $0,50 \mathrm{~m}^{2}$ (Ver Quadro 2). No Módulo Foton, são inseridos os parâmetros de simulação: $o$ parcelamento da abóbada celeste, o qual é escolhido o Globo 8, que se caracteriza por um globo subdividido em 146.198 parcelas $^{1}$; os parâmetros geográficos determinados para a cidade de Florianópolis; a escolha dos tipos de céu predominantes na cidade de estudo: $N^{\underline{o}}$ 2-Céu Encoberto I e $N^{\underline{o}}$ 12-CIE Padrão Céu Claro I, que fazem parte da lista

${ }^{1}$ É o número de parcelas que corresponde ao número de ângulos sólidos em que é dividido o globo. A divisão do Globo vai de 1 até 8; quanto maior seja o Globo, maior a resolução dos resultados nos gráficos. 
validada pelo programa; e finalmente, os parâmetros temporais que correspondem aos meses finais das estações de verão e inverno: 15Março e 15Agosto; e duas horas representativas no horário escolar, de manhã e de tarde: 9:00 horas e 15:00 horas (Ver Quadro 2). Os parâmetros foram aplicados nas 4 orientações principais: Norte, Leste, Sul e Oeste com os 4 tipos de agrupamentos em cada modelo, resultando num total de 128 simulações.

\section{Quadro 2 - Parâmetros inseridos no APOLUX}

\begin{tabular}{rcc}
\multicolumn{4}{|c}{ Parâmetros no Modelo FRACTAL } \\
$\begin{array}{rcc}\text { Layers } & \begin{array}{c}\text { Cor } \\
\text { CAD }\end{array} & \text { Fraccio. }\end{array}$ Área Máx.Fracci. \\
\hline Paredes & 41 & 8 \\
\hline Vidro & 130 & 1 \\
\hline Piso & 254 & 1 \\
\hline Moveis & 9 & 20 \\
\hline lano de Análise & 230 & 20 \\
\hline Alunos & $41 / 160 / 154$ & 1 \\
\hline Quadro & 85 & 8 \\
\hline Teto & 253 & 3 \\
\hline Porta & 44 & 1 \\
\hline
\end{tabular}

Fonte: dos autores

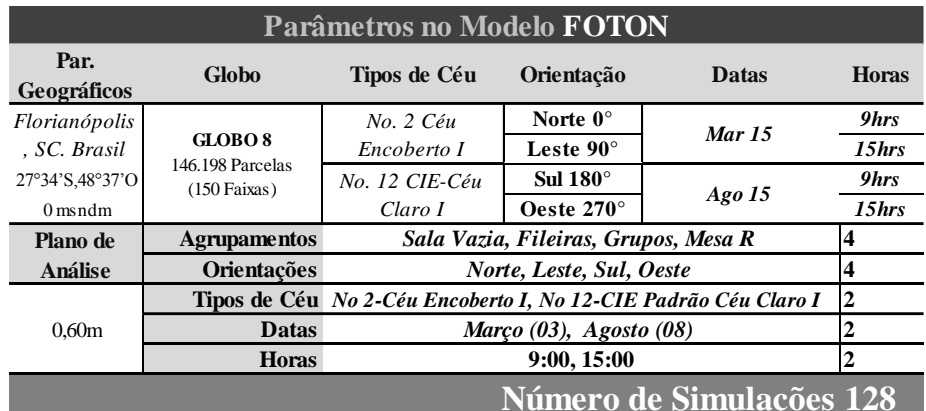

\section{RESULTADOS}

\subsection{Resultados da Simulação Dinâmica}

A seguir são apresentados alguns dados obtidos nas simulações dinâmicas da sala de aula hipotética. A Figura 2, apresenta o mapa de radiação solar, correspondente a um dos modelos nas diferentes orientações com os 4 tipos de agrupamentos, a primeira parte da imagem apresenta, em escala de cores a distribuição da radiação solar $\left(\mathrm{kWh} / \mathrm{m}^{2}\right)$ para a condição Leste_Março_8hrs-12hrs, a segunda parte da imagem informa a percentagem da área do plano de trabalho que encontra-se em cada um dos 3 intervalos de irradiação solar $\left(\mathrm{kWh} / \mathrm{m}^{2}\right)$. Cabe salientar que, para as outras orientações (Norte, Oeste e Sul), foram incluídos nesta figura apenas os dados numéricos mais representativos correspondentes aos percentuais de área por faixa de irradiação.

Os primeiros mapas de radiação foram gerados pelo Plug-in DIVA e a escala de cores falsas foi determinada de acordo com a quantidade mínima e máxima de $\mathrm{kWh} / \mathrm{m}^{2} \mathrm{em}$ todas as simulações. Os segundos mapas foram gerados numa planilha do Excel, onde os dados foram classificados conforme as faixas de $\mathrm{kWh} / \mathrm{m}^{2}$ que foram consideradas como representativas: valores inferiores a $2,0 \mathrm{kWh} / \mathrm{m}^{2}$ (cor amarelada clara), valores entre $2,0 \mathrm{kWh} / \mathrm{m}^{2}$ e $10,0 \mathrm{kWh} / \mathrm{m}^{2}$ (cor laranja), e valores que ultrapassam os $10,0 \mathrm{kWh} / \mathrm{m}^{2}$ (cor laranja escuro). A segunda informação representa as percentagens da área do plano de trabalho da Sala $\left(60,75 \mathrm{~m}^{2}\right)$ em cada faixa de irradiação $\left(\mathrm{kWh} / \mathrm{m}^{2}\right)$ para cada um dos agrupamentos, nas orientações/meses/intervalos de hora. As percentagens foram representadas em quadros de cor cinza que vão desde o cinza mais escuro $(0 \%)$ até o cinza mais claro $(100 \%)$. 
Figura 2 - Mapa de Radiação solar por Agrupamento
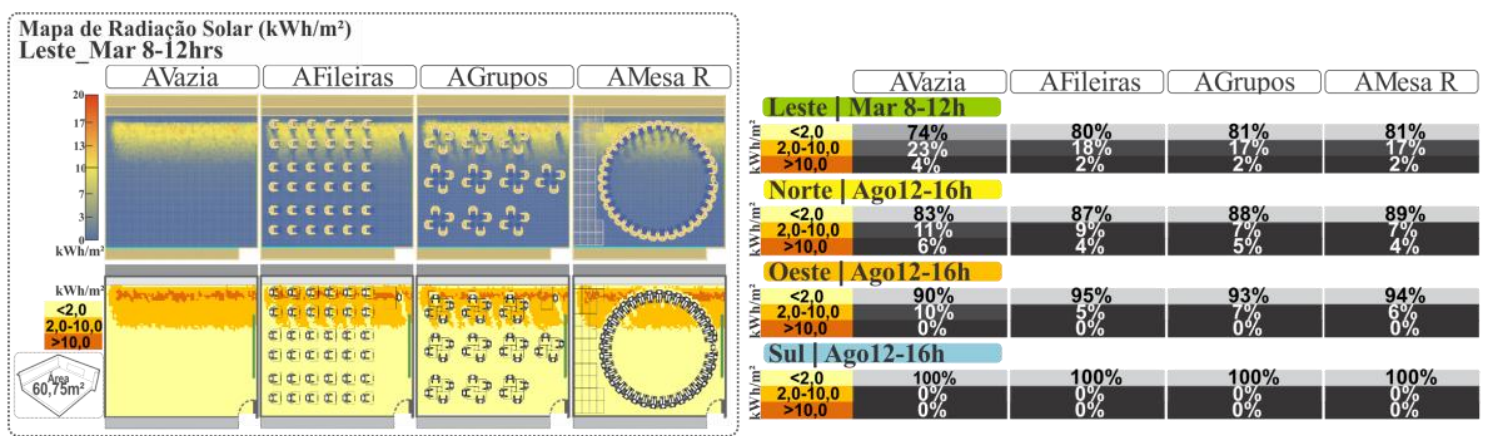

Fonte: dos autores

Como se observa na Figura 2, não se apresenta uma diferença aparente nos mapas de radiação entre os agrupamentos. Contudo os dados numéricos informam que o Agrupamento Sala Vazia (AVazia), apresenta a maior irradiação solar no interior da sala nas orientações/meses/intervalos de hora avaliadas. Para as salas orientadas a Sul nos períodos da tarde, não existe diferença representativa nos valores, já que em todos os agrupamentos, os valores são inferiores a $2,0 \mathrm{kWh} / \mathrm{m}^{2}$.

A seguir se apresenta um gráfico de colunas com os dados numéricos das salas orientadas a Leste e Norte da Figura 2. Nos gráficos da Figura 3, se ilustra a diferença percentual em cada faixa dos agrupamentos com a presença de estudantes comparada com o agrupamento Sala Vazia.

\section{Figura 3 - Diferença percentual entre agrupamentos com estudantes e agrupamento Sala Vazia}

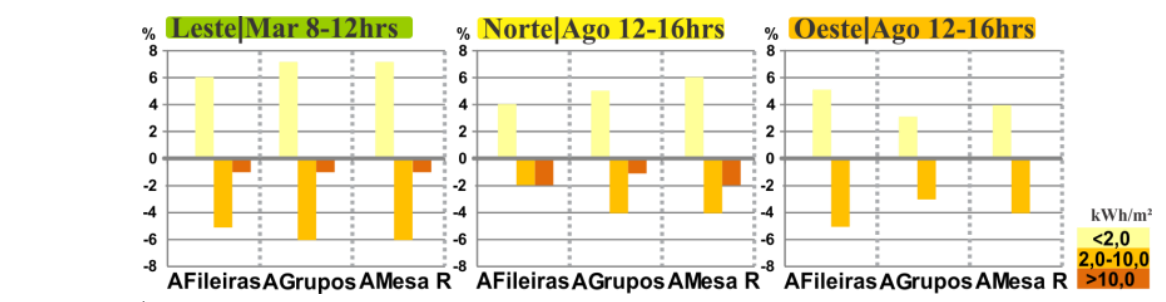

Fonte: dos autores

A diferença percentual indica que nos valores inferiores a $2,0 \mathrm{kWh} / \mathrm{m}^{2}$ as percentagens dos agrupamentos aumentam em relação as percentagens do agrupamento Sala Vazia em nas três orientações: no Leste, em $6 \%$ no agrupamento AFileiras até $7 \%$ no agrupamento AGrupos e AMesa $R$, no Norte, em $4 \%$ no agrupamento AFileiras até em $6 \%$ no agrupamento AMesa $R$ e no Oeste, em 4\% no agrupamento AMesa $R$ até $5 \%$ no agrupamento AFileiras. Estas percentagens são diminuídas principalmente na faixa dos valores entre $2,0 \mathrm{kWh} / \mathrm{m}^{2}$ e $10,0 \mathrm{kWh} / \mathrm{m}^{2}$. O anterior demostra que a presença dos estudantes aumenta a percentagem da área do plano de trabalho na faixa dos valores inferiores aos 2,0 kWh/m² e diminui nas faixas maiores afirmando que a ocupação interfere no ingresso da irradiação na Sala.

A Figura 4 apresenta dois gráficos de colunas para os meses de Março e Agosto: o primeiro representa a Média dos valores Máximos de irradiação solar $\left(\mathrm{kWh} / \mathrm{m}^{2}\right)$ e o segundo representa a Média dos valores Intermediários de irradiação solar $\left(\mathrm{kWh} / \mathrm{m}^{2}\right)$. Foram gerados ambos os gráficos já que entre as médias dos valores intermediários e máximos se apresentam variações muito grandes. 


\section{Figura 4 - Irradiação Solar por Orientação $\left(\mathbf{k W h} / \mathrm{m}^{2}\right)$}

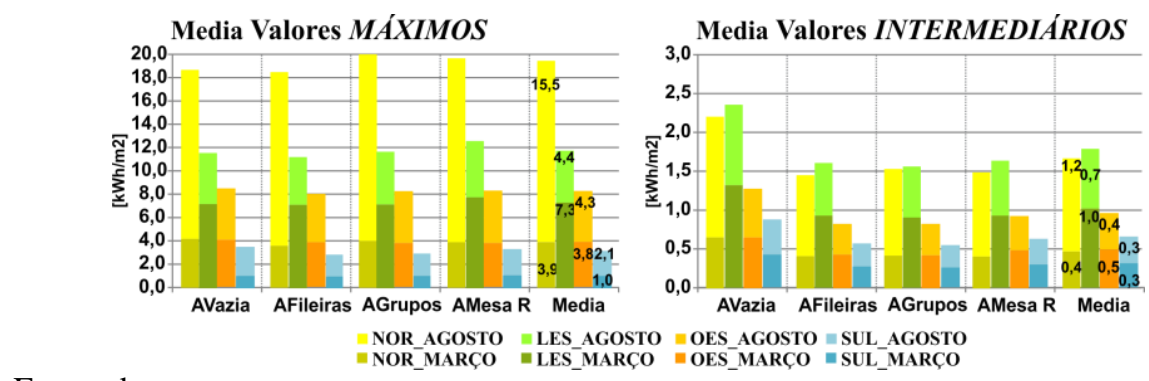

Fonte: dos autores

No gráfico da Média dos valores Máximos os maiores valores de irradiação solar ocorrem na orientação Norte, no mês de Agosto, nas salas com os agrupamentos AMesarR $\left(16,56 \mathrm{kWh} / \mathrm{m}^{2}\right)$ e AGrupos $\left(16,01 \mathrm{kWh} / \mathrm{m}^{2}\right)$. Contudo, no gráfico da média dos valores Intermediários o maior valor de irradiação solar ocorre na mesma orientação e data, na Sala Vazia com valores ligeiramente superiores $\left(1,55 \mathrm{kWh} / \mathrm{m}^{2}\right)$ aos dos outros agrupamentos. Por outro lado, no gráfico da Média dos valores Máximos, os menores valores de irradiação solar foram apresentados na orientação Sul, no mês de Março, nas salas com os agrupamentos AMesar $\mathrm{R}\left(1,09 \mathrm{kWh} / \mathrm{m}^{2}\right)$ e AGrupos $(1,108$ $\mathrm{kWh} / \mathrm{m}^{2}$ ). Contudo, na avaliação da Média dos valores Intermediarios os menores valores de irradiação solar ocorrem na mesma orientação e data, nas salas com os agrupamentos AGrupos $\left(0,22 \mathrm{kWh} / \mathrm{m}^{2}\right)$ e AFileiras $\left(0,29 \mathrm{kWh} / \mathrm{m}^{2}\right)$.

\subsection{Resultados da Simulação Estática}

A seguir serão apresentados os dados obtidos nas simulações estáticas da sala de aula hipotética para condições de Céu Claro. Nesta condição se percebem melhor as mudanças na distribuição da luz. A Figura 5 apresenta a distribuição das iluminâncias nos diferentes agrupamentos em gráficos 3D para a orientação Leste_Março_9hr. Foram incluídos os dados numéricos para Oeste_Agosto_15hrs já que apresentam comportamentos semelhantes a Leste_Março_9hrs.

\section{Figura 5 - Distribuição das Iluminâncias por Área nos Agrupamentos}

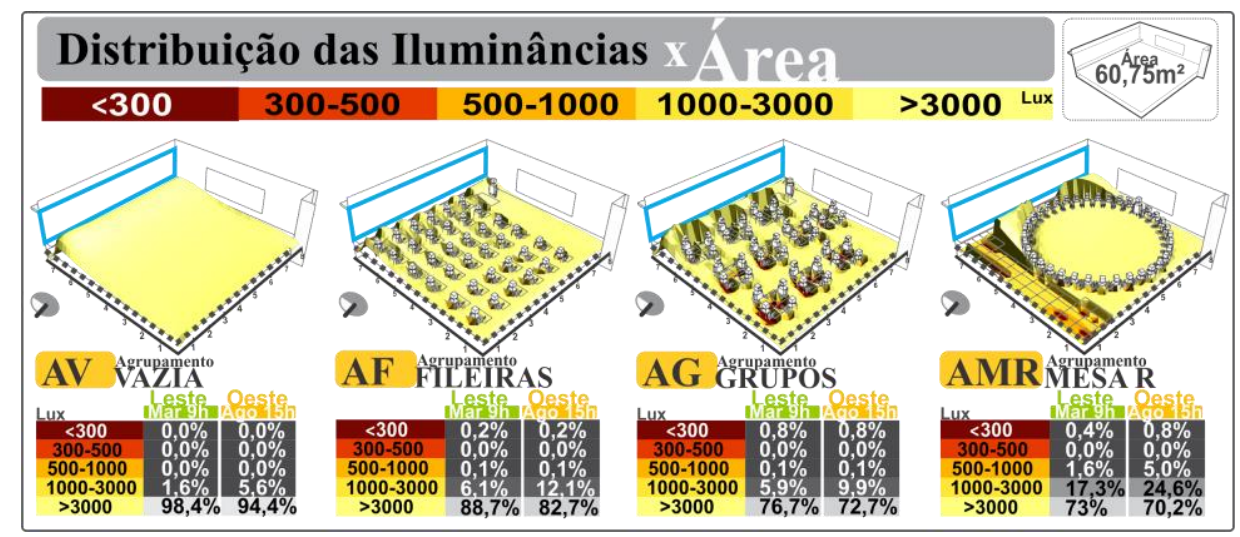

Fonte: dos autores

A distribuição das iluminâncias é classificada em percentagens da área total da sala $\left(60,75 \mathrm{~m}^{2}\right)$ que se encontram dentro de cada intervalo (faixa). A classificação das faixas foi determinada conforme aos valores mínimos e máximos na distribuição da luz. As 
faixas não foram determinadas com as exigências de conforto visual descritas pelas normas, por isso não se aprofundou em conceitos de desejabilidade de níveis de iluminação por parte dos usuários no presente estudo. As faixas foram estimadas da seguinte forma: faixa baixa (cor vermelha escuro), área onde se encontram os estudantes/móveis, com valores inferiores a 300 lux; faixa intermediária (cor vermelha clara), área de trabalho dos estudantes (escrivaninhas), com valores entre 300 lux e 500 lux (nível requerido segundo a norma); faixa alta (cor laranja e amarela clara), área de trabalho dos estudantes, com valores de 500 lux a 1000 lux (nível máximo segundo a norma) e área da circulação dos estudantes com valores entre os 1000 lux e 3000 lux; faixa excessiva (cor amarelada clara), área perto da janela ou sem estudantes com valores superiores a 3000 lux.

Como se observa na Figura 5, o agrupamento Sala Vazia (AVazia), apresenta os maiores níveis de iluminação, com quase 98,4\% (Leste_Mar_9hrs) e 94,4\% (Oeste_Ago_15hrs) da área da sala de aula com valores que aumentam em relação a 3000 lux, faixa que caracteriza as elevadas iluminâncias perto da janela e a ausência de estudantes. Nos demais agrupamentos, as diferenças percentuais da área na faixa excessiva com o agrupamento Sala Vazia (AVazia) se apresentam com valores levemente inferiores: para Leste_Mar_9hrs, as diferenças estão entre 9,7\% (AFileiras) até 25,4\% (AMesa R); e Oeste_Ago_15hrs, diferenças entre 11,7\% (AFileiras) até $24,2 \%$ (AMesa R).

Em ambos os casos os valores mais baixos se apresentam no agrupamento Afileiras e os mais altos no agrupamento AMesa $R$, se apresente maior percentagen de área principalmente entre 1000 lux e 3000 lux. Os agrupamentos AFileiras e o Agrupos apresentam comportamentos similares na distribuição das iluminâncias comparados com a Sala Vazia nas diferentes faixas.

Com relação o AMesa $R$ embora mostra aumentos nas faixas altas, as proporções diferem dos outros agrupamentos, com valores de $17,3 \%$ e $24,6 \%$ da área da sala na faixa entre 1000 lux e 3000 lux. Este comportamento pode estar sendo causado pela forma e concentração das escrivaninhas neste agrupamento. A Figura 6 mostra 4 gráficos de colunas com a curva de distribuição das iluminâncias nas condições avaliadas com os valores maiores e mais representativos de todas as simulações feitas. A curva foi gerada a partir da seção transversal da Sala $(4,4 \mathrm{~m})$ para os 4 agrupamentos.

\section{Figura 6- Curva de Distribuição das Iluminâncias por Agrupamentos}

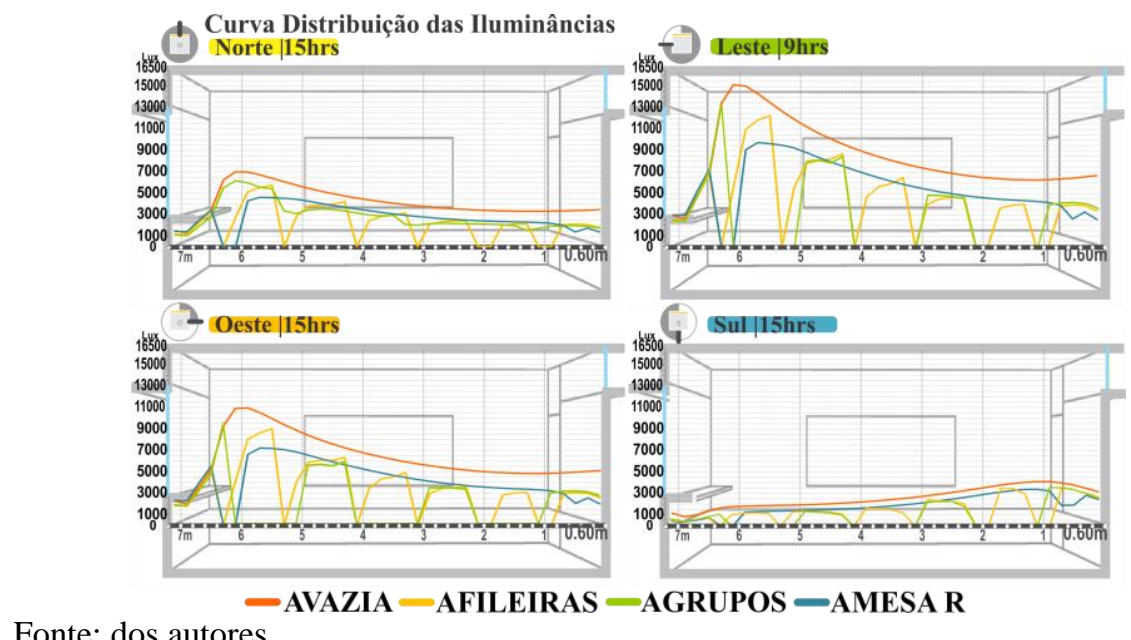


De acordo com a Figura 6, existem variações na distribuição da luz e nos valores de iluminância, conforme as datas e horários. Na orientação Norte, ocorrem os maiores níveis das iluminâncias durante o mês de Agosto, as 15hrs, próximo à janela; já no Leste, os maiores níveis das iluminâncias em Março e Agosto ocorrem as 9hrs, também perto da janela; no Oeste, a ocorrência dos maiores níveis também se dá as $15 \mathrm{hrs,} \mathrm{perto}$ da janela e no Sul, os maiores níveis ocorrem no mês de Agosto, as 15hrs, no fundo da sala.

Como se observa, a curva da distribuição das iluminâncias da sala Vazia está acima das curvas dos outros agrupamentos em todas as orientações/horários. Nessa mesma ordem a curva do agrupamento Afileiras apresenta um comportamento similar ao do agrupamento AGrupos: os picos mínimos correspondem a presença dos estudantes e os picos máximos correspondem aos níveis das iluminâncias maiores, com a sua distribuição parcialmente influenciada pela ocupação. No caso do agrupamento AMesa $R$, embora a curva seja levemente inferior a da Sala Vazia, a tendência da mesma é decrescer de forma menos abrupta do que os outros agrupamentos com estudantes.

\section{CONCLUSÕES}

Conforme aos resultados obtidos das simulações dinâmicas se permite concluir que a tendência dos agrupamentos é que Sala Vazia apresente maior irradiação em comparação com os demais agrupamentos em todas as orientações, inclusive no menor ingresso da irradiação. Além disso, na media dos valores Intermediarios, o agrupamento AVazia e AMesa $R$ apresentam os valores maiores.

Isto permite afirmar que a ocupação e a forma como é ocupada a sala influi na irradiação. Embora, existe presença de estudantes no agrupamento AMesa $R$, eles se encontram próximos uns de outros ocupando pouca área da Sala. Portanto são maiores os ingressos de irradiação em aqueles agrupamentos onde os estudantes se encontram afastados e dispersos pela Sala. Quer dizer, quanto menor a área ocupada pelos estudantes, maior a presença de irradiação no interior da Sala.

Cabe salientar que o estudo da irradiação solar no plano de trabalho permite, em futuros trabalhos, reconhecer as zonas da sala onde existe maior influencia da irradiação solar, realizar estudos de conforto térmico, no quais o usuário interage nas condições da sala, por exemplo, fechando as cortinas ou mudando de lugar. Todos estes aspectos não foram aprofundados no presente estudo.

Com relação aos resultados obtidos das simulações estáticas se permite concluir que continua a mesma tendência da incursão solar: a Sala Vazia apresenta um crescimento maior na curva da distribuição das iluminâncias em comparação com a curva dos outros agrupamentos nas condições avaliadas, o que permite confirmar mais uma vez a influencia da ocupação na distribuição das iluminâncias na sala. A distribuição das iluminâncias da Sala Vazia e o agrupamentos AMesa $R$ apresentam comportamentos similares em relação ao crescimento da curva: ambas decrescem de forma continua à medida que se afastam da janela. Por sua parte, as curvas das iluminâncias dos agrupamentos AFileiras e AGrupos decrescem de acordo à influencia da ocupação. A mesma tendência da incursão solar ocorre na distribuição das iluminâncias: quanto mais próximos os estudantes que corresponde a menor área ocupada, maior a distribuição das iluminâncias no interior da Sala. 
Conforme ao anterior, podem se estimar vocações de uso da sala de aula, onde se otimize a área total da sala por agrupamentos e atividades de acordo com a época do ano e o período horário. Por exemplo, considerar agrupamentos mais próximos nas manhãs do inverno e mais afastados nas tardes do verão.

Finalmente com os resultados das curvas do Norte, Oeste e Leste, além da confirmação da influencia da ocupação, pode existir um zoneamento da Sala de aula estimado da seguinte forma: Zona 1 (perto da janela, 2,50m), onde a curva da distribuição atinge o pico maior dentro da Sala; Zona 2 (no meio da Sala, 2,50m), onde a curva da distribuição atinge o pico menor com diferenças nas iluminâncias, por exemplo na Sala Vazia de 2500 lux (Norte), 4500 lux (Leste) e 6000 lux (Oeste); e Zona 3 (fundo da Sala, 2,50m), onde a curva tende se estabilizar, inclusive as diferenças entre os agrupamentos são levemente reduzidas. $\mathrm{O}$ anterior permite concluir que a Zona 2 é a zona com maiores diferenças entre uma zona com os picos máximos da luz e uma zona com a luz mais estável.

Neste estudo sobre a influencia da ocupação na distribuição da luz e em consequência a classificação das zonas da Sala pode servir como método de análise para outros espaços com qualidades que possuam qualidades de permanência significativas e que necessitem atingir exigências luminosas básicas.

\section{AGRADECIMENTOS}

À CAPES, pela bolsa de estudos, ao Pós-ARQ e ao LabCon/ARQ- UFSC, pelo apoio recebido.

\section{REFERÊNCIAS}

ASSOCIAÇÃO BRASILEIRA DE NORMAS TÉCNICAS - ABNT. ABNT NBR ISO/CIE 8995-1:2013. Iluminañção de ambientes de trabalho Parte 1:Interior. Rio de Janeiro, RJ: [s.n.]. , 2013

AZEVEDO, G. A. N. Arquitetura Escolar e Educação: Um Modelo Conceitual de abordagem interacionalista. 2002. Universidade Federal do Rio de Janeiro, COPPE/UFRJ, 2002.

FOTIOS, S. A.; RAMASOOT, T. Lighting for the Classrooms of the Future. Electronic classrooms: a new challenge for school lighting guidance. Light \& Engineering 17 (2). School of Architecture, University of Sheffield, UK, p. 62-70, 2009.

FUNDAESCOLA, F. DE F. DA E. M. DE E. Espaços Educativos Ensino fundamental subsídios para Elaboração de Projetos e adequação de edificações escolares. Cadernos Técnicos 4 - Volume 1. Brasilia: [s.n.], 2002.

HATHAWAY et al. A Study Into the Effects of Light on Children of Elementary School Age - A Case of Daylight Robbery. Alberta, Canadá: Policy and Planning Branch Department, Planning and Information Services Division. , 1992

HESCHONG MAHONE GROUP. Daylighting in Schools: An Investigation into the Relationship between Daylighting and Human Performance. Detailed Report. . California: [s.n.], 1999.

IBAM_INSTITUTO BRASILEIRO DE ADMINISTRAÇÃO MUNICIPAL. Manual para Elaboração de Projetos de Edifícios Escolares na Cidade do Rio de Janeiro. Rio de Janeiro: IBAM/CPU, PCRJ/SMU: [s.n.]. , 1996

MEN COLOMBIA. Norma Técnica Colombiana NTC 4595 Ingeniería Civil y Arquitectura. Planeamiento y Diseño de Instalaciones y Ambientes Escolares. [S.l: s.n.]. Disponível em: <www.mineducacion.gov.co>. , 2006 